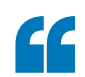

Pax2 null cells failed to differentiate into nephron cells [and] underwent a cell-fate switch

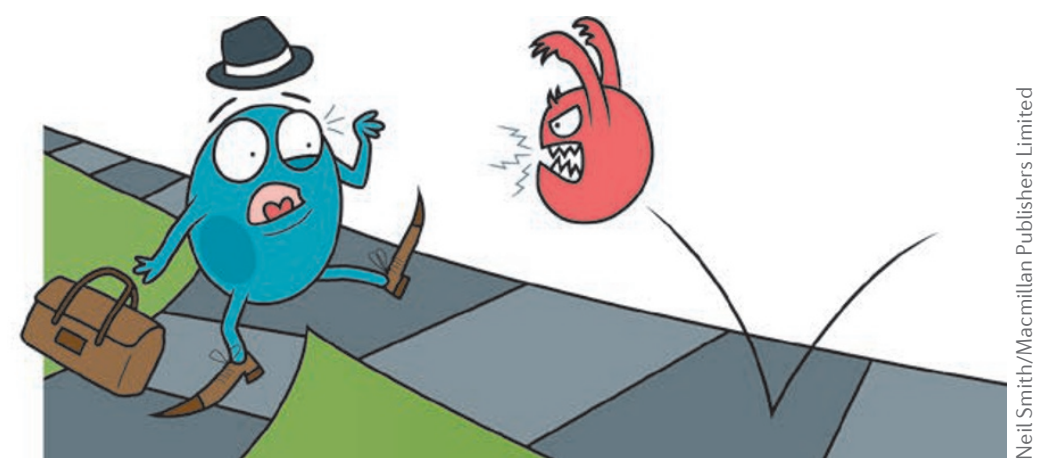

DEVELOPMENT

\section{Pax2 keeps nephron progenitors on track}

Low nephron numbers can increase susceptibility to chronic kidney disease.

Nephrons and the surrounding interstitial tissue arise from two distinct progenitors that are separated by a lineage boundary. The molecular determinants of this fate barrier, which is largely maintained in healthy, diseased and regenerating kidneys, are currently unclear. A new study conducted by Akio Kobayashi and co-workers shows that Pax2 establishes the boundary between nephron and interstitial lineages during kidney organogenesis by repressing the interstitial fate of nephron progenitors.

The complete ablation of $\mathrm{Pax} 2$, a key regulatory factor in renal development, stunts kidney organogenesis at an early stage and results in kidney agenesis, which has prevented studies on the function of $\mathrm{Pax} 2$ in specific tissues. To circumvent this issue, the researchers ablated Pax2 exclusively in the cap mesenchyme, the nephron progenitors, in a new mouse model.

In these mice, the Pax2-deficient cap mesenchyme was successfully generated but this cell population was lost within 2 days. Genetic cell-fate tracing showed that Pax2 null cells fail to differentiate into nephron cells, instead undergoing a cell-fate switch and transdifferentiating into renal interstitial progenitors and inner renal medullary interstitial cells. Single-cell RNA-Seq profiling revealed that Pax2-deficient cells clustered with interstitial cells and adopted an interstitial gene expression signature. They upregulated interstitial markers such as Meis1, Anxa2 and Foxd1 and downregulated cap-mesenchyme markers such as Six2, Crym and Eya1. Thus, Pax2 maintains nephron progenitor identity by suppressing activation of the interstitial programme and preventing transdifferentiation into interstitial cells.

To investigate whether repression of the interstitial cell fate occurs in a cell-autonomous fashion in the cap mesenchyme, the investigators performed mosaic ablation of $\mathrm{Pax} 2$ in nephron progenitors. Pax2 deletion in some nephron progenitors was not compensated for by surrounding $\mathrm{Pax}^{+}$cells and induced loss of the cap mesenchyme identity, indicating that Pax2 cell-autonomously represses interstitial fate in nephron progenitors.

This novel understanding of the involvement of $\mathrm{Pax} 2$ in the regulation of nephron formation will shed light on how a full set of functional nephrons arises and could help develop therapeutic strategies to restore nephron endowment in conditions that reduce nephron numbers such as premature birth and malnutrition.

Andrea Aguilar

ORIGINAL ARTICLE Naiman N. et al. Repression of interstitial identity in nephron progenitor cells by Pax2 establishes the nephronlnterstitium boundary during kidney development. Dev. Cell http://dx.doi.org/ 10.1016/j.devcel.2017.04.022 (2017)

FURTHER READING Little, M. H. et al. Understanding kidney morphogenesis to guide renal tissue

regeneration. Nat. Rev. Nephrol. 12, 624-635 (2016) 